\title{
Spectroscopic Rationale for Efficient Stimulated-Emission Depletion Microscopy Fluorophores
}

\author{
Jun-ichi Hotta, ${ }^{, \dagger}$ Eduard Fron, ${ }^{\dagger}$ Peter Dedecker, ${ }^{\dagger}$ Kris P. F. Janssen, ${ }^{\ddagger}$ Chen Li, ${ }^{\S}$ Klaus Müllen, ${ }^{\S}$ \\ Benjamin Harke," Johanna Bückers," Stefan W. Hell," and Johan Hofkens ${ }^{\dagger}$ \\ Department of Chemistry, Katholieke Universiteit Leuven, Celestijnenlaan 200F, B-3001 Heverlee, Belgium, \\ BIOSYST - MeBioS, Katholieke Universiteit Leuven, Willem de Croylaan 42, B-3001 Heverlee, Belgium, \\ Max-Planck-Institut für Polymerforschung, Ackermannweg 10, 55128 Mainz, Germany, and Department of \\ NanoBiophotonics, Max Planck Institute for Biophysical Chemistry, Am Fassberg 11, 37077 Göttingen, Germany
}

Received January 5, 2010; E-mail: junichi.hotta@chem.kuleuven.be

For decades, the power of far-field fluorescence microscopy has been hampered by the diffraction resolution limit, ${ }^{1,2} d=\lambda /(2 \mathrm{NA})$ $>200 \mathrm{~nm},{ }^{3,4}$ where $\lambda$ denotes the light wavelength and NA the numerical aperture of the lens. Identical fluorophores closer than $d$ cannot be discerned because their signals cannot be separated by the detector. However, in the recent past, it has been demonstrated that the diffraction limit can be fundamentally overcome ${ }^{5}$ by modulating or switching the ability of the dye to emit fluorescence, causing adjacent objects to be registered sequentially. To this end, stimulated-emission depletion (STED) microscopy ${ }^{5,6}$ and its derivatives modulate the fluorescence capability of dye ensembles using defined spatial patterns of light, whereas the techniques known as (f)PALM, ${ }^{7,8}$ (d)STORM,,${ }^{9,10}$ and related concepts ${ }^{11}$ involve switching individual fluorophores stochastically in space followed by mathematical localization of their coordinates. Hence, all of these concepts harness a molecular mechanism that keeps the dye nonresponsive when it is exposed to excitation light.

In STED microscopy (Figure 1), ${ }^{12}$ the dyes are kept nonresponsive (dark) by subjecting them to red-shifted light, called the STED beam, which induces stimulated emission from their fluorescent state, $S_{1}$, to their ground state, $S_{0}$. The maximum occupation probability of the $S_{1}$ state decreases nearly exponentially with the intensity $I$ of the STED beam. Applying the condition $I$ $\gg I_{\mathrm{s}}$, where $I_{\mathrm{s}}$ is a dye constant, virtually confines the dye to the dark $\mathrm{S}_{0}$ state, even if it is subject to excitation light. Usually formed as a donut, the STED beam is superimposed upon the regularly focused excitation spot to keep dark all of the dye molecules that are potentially present except those at the center of the donut. Only dyes that happen to be located within half the distance from the center

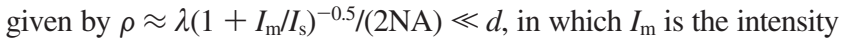
at the donut crest, are allowed to be bright ( $\rho$ is the resolution in STED). Scanning the superimposed beams across the object sequentially registers fluorophores that are further than $\rho$ apart. The resolution $\rho$ can in principle become arbitrarily high for $I \gg I_{\mathrm{s}}$. Clearly, the fluorophores that had been kept dark by STED must instantly become bright when they fall into the donut minimum, but this is the case only if they have not been transferred to a long-lived dark state. This aspect is critical for small $\rho$, because larger values of $I$ increase the probability that a dye in the $S_{1}$ state passes to such a long-lived dark state, or simply bleached. In contrast, stimulated emission is instant, reversible, and so fundamental that almost any fluorophore can be transiently turned off by a STED beam. ${ }^{13}$ Thus, if long-term

\footnotetext{
${ }^{\dagger}$ Department of Chemistry, Katholieke Universiteit Leuven.

* BIOSYST - MeBioS, Katholieke Universiteit Leuven.

\& Max-Planck-Institut für Polymerforschung.

"Max Planck Institute for Biophysical Chemistry.
}

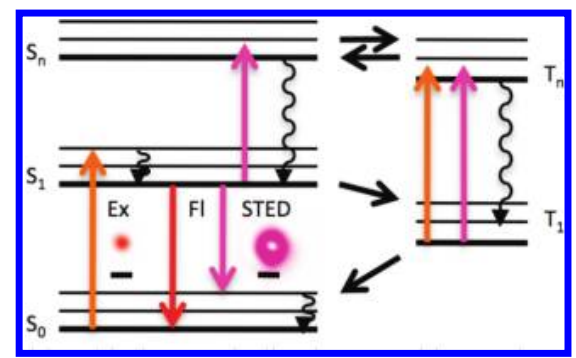

Figure 1. Jablonski diagram indicating transitions relevant for STED. Scale bars under the excitation and focal spots represent $500 \mathrm{~nm}$.

darkening is minimized, STED should provide nanoscale resolution on the ensemble level with nearly any fluorophore.

Therefore, avoiding any process that competes with stimulated emission is prudent. Tuning the STED beam wavelength to the red edge of the fluorescence spectrum largely avoids excitation from the $S_{0}$ state, but transitions originating from excited states may still occur, such as crossing to the triplet states, $\mathrm{T}_{n}$, as well as absorption to $\mathrm{S}_{n}$ and $\mathrm{T}_{n}$ states with $n>1$ (Figure 1). In fact, many bleaching pathways involve triplet states, ${ }^{14,15}$ which are effectively populated through the $S_{n}$ states. The higher $S_{n}$ states can also lead to bleaching directly. ${ }^{16}$ Both processes compete with favorable ultrafast nonradiative $\mathrm{S}_{n} \rightarrow \mathrm{S}_{1}$ (or $\mathrm{T}_{n} \rightarrow \mathrm{T}_{1}$ ) relaxation followed by spontaneous or stimulated emission. ${ }^{13}$

With these conditions in mind, we explored the use of rylene dyes for STED microscopy. In view of their large extinction coefficient, brightness, and photostability (especially when applied in materials sciences ${ }^{4,17}$ ), these dyes should, at face value, be ideal for STED. Specifically, we investigated tetraphenoxy-substituted perylenediimide (pPDI) and tetraphenoxy-substituted terylenediimide (pTDI) ${ }^{18}$ dyes, which have revealed remarkable photostability and low intersystem crossing in single-molecule experiments. ${ }^{17}$ Single pPDI and pTDI molecules can be registered for over $1 \mathrm{~h}$ without photobleaching and with few excursions to dark states. ${ }^{17}$

STED microscopy has been carried out with excitation light pulses followed by pulses for STED or with continuous wave (CW) beams for excitation and STED. The pulsed-mode setup has been reported elsewhere, ${ }^{6,19}$ while the $\mathrm{CW}$ system used here is detailed in the Supporting Information. First attempts with pPDI in the CW mode using a STED wavelength of $700 \mathrm{~nm}$ exhibited pronounced bleaching, as only $<40 \%$ of the molecules seen in the confocal image were also found in the STED image (Figure 2a,b). Using 76 $\mathrm{MHz}$ pulse pairs with a STED wavelength of $740 \mathrm{~nm}$ slightly reduced the bleaching; a resolution gain by a factor of $\sim 3$ was observed ( $\sim 65 \mathrm{~nm}$; see Figure S2 in the Supporting Information) when a STED power of $33 \mathrm{~mW}$ was applied in the focal plane. 


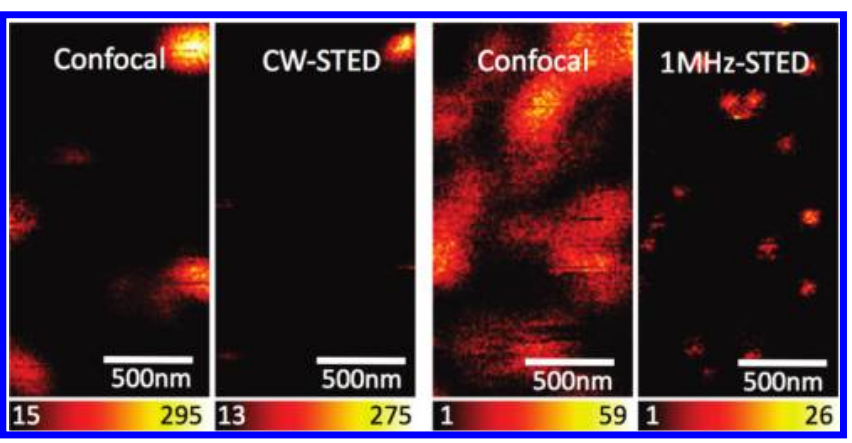

Figure 2. CW-STED image of pPDI exhibiting bleaching. In the CW mode, the excitation $(543 \mathrm{~nm})$ and STED $(700 \mathrm{~nm})$ beam powers were $2.0 \mu \mathrm{W}$ and $16 \mathrm{~mW}$, respectively. Bleaching was slightly reduced upon application of pulsed-mode STED microscopy with a $1 \mu$ s interpulse time interval using a $2.0 \mu \mathrm{W}(543 \mathrm{~nm})$ excitation beam and a $670 \mu \mathrm{W}(710 \mathrm{~nm})$ STED beam. The pixel dwell times for each pixel were 2 and $1 \mathrm{~ms}$ for $\mathrm{CW}$ and $1 \mathrm{MHz}$ pulsed STED, respectively.
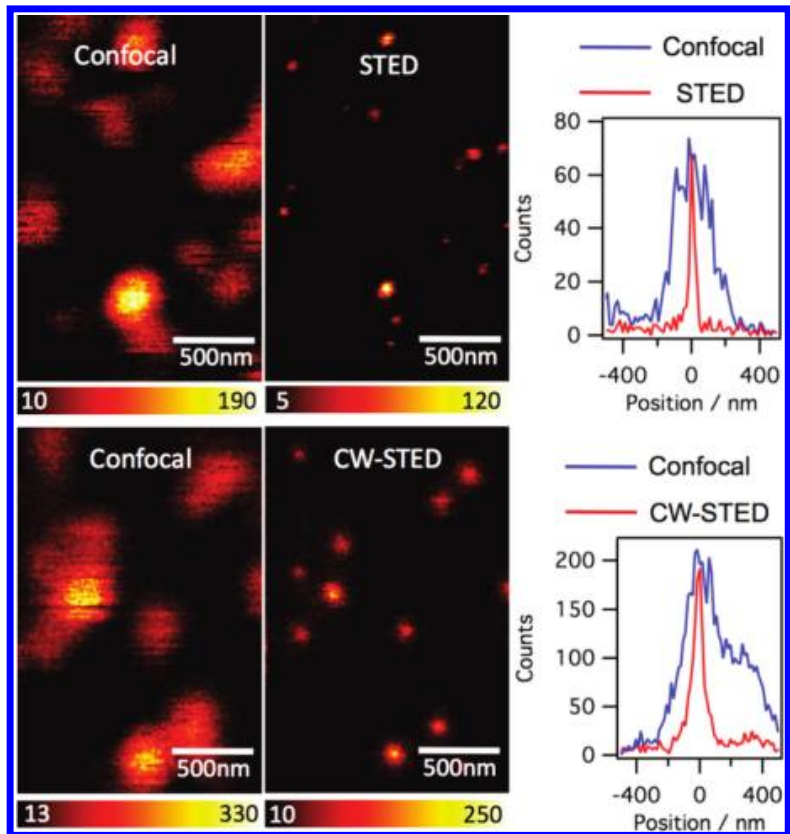

Figure 3. Confocal vs STED images of single pTDI molecules for (top) $76 \mathrm{MHz}$ pulsed STED and (bottom) CW-STED. The bin times for each pixel were 0.5 and $2 \mathrm{~ms}$ for the pulsed and CW-STED modes, respectively. In the pulsed mode, the average excitation power was $4.0 \mu \mathrm{W}(635 \mathrm{~nm})$, whereas in the $\mathrm{CW}$ mode, $2.5 \mu \mathrm{W}(633 \mathrm{~nm})$ was employed. The average power of the pulsed STED beam $(775 \mathrm{~nm})$ was $32 \mathrm{~mW}$, whereas that of the CW-STED beam $(780 \mathrm{~nm})$ was $58 \mathrm{~mW}$. The fwhm of pulsed STED and CW-STED images of single molecules (point spread functions) were 34 and $78 \mathrm{~nm}$, respectively.

For this power setting, $\sim 50 \%$ of the molecules could be observed after acquisition of the STED image. Using a lower repetition rate of $1 \mathrm{MHz}$ reduced the bleaching to some extent, since the associated time between the pulse pairs $(1 \mu \mathrm{s})$ allowed relaxation of dark states before the arrival of the succeeding pulse pair ${ }^{15,20}$ and gave slightly better resolution (55 nm; see Figure S3). In contrast, as shown in Figure 3, pTDI exhibited excellent performance at $76 \mathrm{MHz}$, with $\sim 95 \%$ of the molecules in the confocal image also emerging in the STED counterpart. The resolution obtained with the employed STED beam power was $35 \mathrm{~nm}$ (without further data treatment). pTDI showed equally good performance in the $\mathrm{CW}$ mode too, with $90 \%$ recovery and $75 \mathrm{~nm}$ resolution. Longer interpulse breaks were not required. ${ }^{20}$

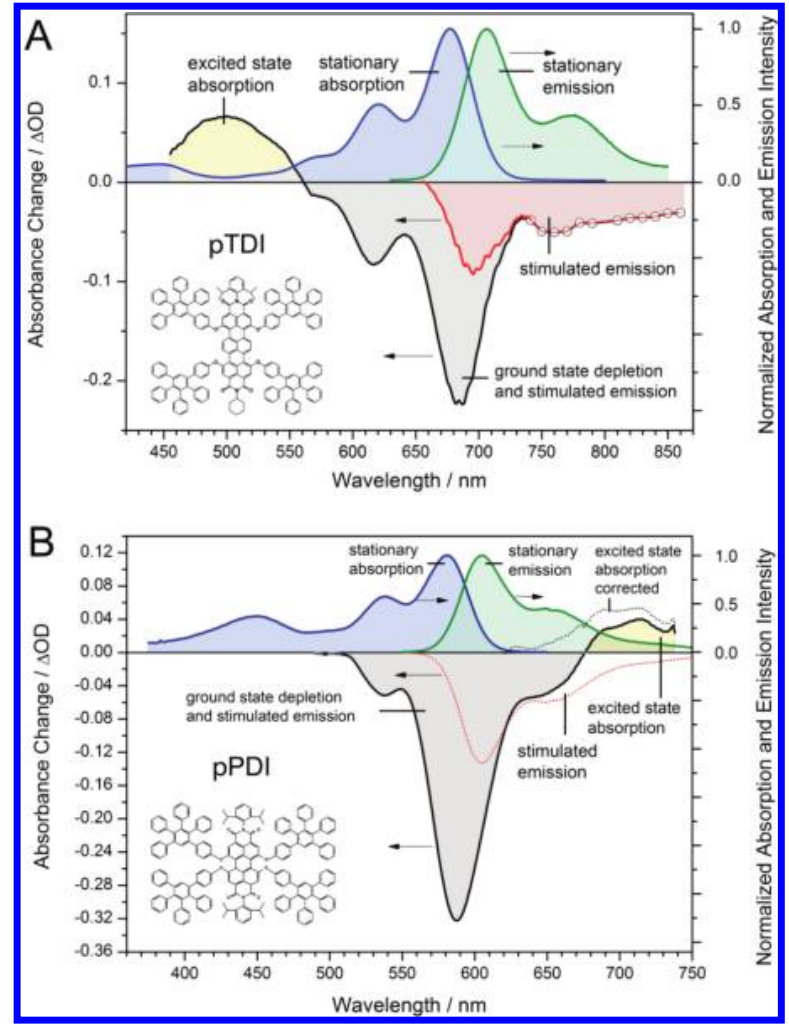

Figure 4. Transient absorption spectra of pTDI and pPDI. The transient absorption band $\left(\mathrm{S}_{1} \rightarrow \mathrm{S}_{n}\right)$ is blue-shifted for pTDI and well-separated from the stimulated-emission band and STED de-excitation beam wavelength $(780 \mathrm{~nm})$ used. The excited-state absorption band of pPDI overlaps with the stimulated-emission spectrum; at the STED beam wavelength $(700 \mathrm{~nm})$, strong $\mathrm{S}_{1} \rightarrow \mathrm{S}_{n}$ absorption occurs.

Since in the reported single-molecule (excitation only) experiments the photostability of pPDI was comparable to that of pTDI, we found the differences observed with STED rather puzzling. We therefore carried out femtosecond transient absorption measurements to elucidate the photophysics and photochemistry of the excited states of the two fluorophores. ${ }^{21}$

Figure 4 shows the transient absorption spectra recorded $5 \mathrm{ps}$ after excitation for pPDI and pTDI in toluene. In these plots, augmented optical density indicates absorption of the $S_{1}$ state populated after excitation, while negative values are due to a superposition of ground-state depletion (the disappearance of the $\mathrm{S}_{0}$ absorption due to the formation of an excited state) and stimulated emission from the $S_{1}$ state.

Both pPDI and pTDI show strong negative contributions originating from ground-state depletion and stimulated emission. More interesting, however, are their $\mathrm{S}_{1} \rightarrow \mathrm{S}_{n}$ absorption bands. In the case of pTDI, this band is blue-shifted relative to the $S_{0} \rightarrow S_{1}$ absorption. In contrast, pPDI exhibits $S_{1} \rightarrow S_{n}$ absorption at the red edge of the emission spectrum.

To avoid anti-Stokes excitation by the STED beam, its wavelength is typically chosen to lie in the red tail of the emission spectrum. For pTDI, this is a safe choice that leads to the excellent STED performance demonstrated above. Altogether, these results indicate that a good STED dye shows negligible $S_{1} \rightarrow S_{n}$ absorption at the STED wavelength, thereby avoiding population of higher excited states.

In the case of pPDI, the situation is different. While stimulated emission undoubtedly occurs, the fluorophore can effectively absorb a photon and move on to a higher-energy $S_{n}$ state. The fluorophore can rapidly (within $<5 \mathrm{ps}$ ) convert back to the $\mathrm{S}_{1}$ state, from which 
it fluoresces, undergoes stimulated emission, or re-enters the same cycle, each with a probability corresponding to the transition probability of the respective process. Unfortunately, non-negligible excited-state absorption fosters repeated excitation to the $S_{n}$ state and hence photobleaching, either by facilitating crossing to the $\mathrm{T}_{n}$ state or by starting a bleaching reaction straight from the $S_{n}$ state. ${ }^{21}$ Clearly, the average number of cycles into higher excited states per fluorophore increases with smaller $\rho$.

The deconvolution of the transient absorption spectrum (500-740 $\mathrm{nm}$ ) into the corresponding ground-state absorption, stimulatedemission, and excited-state absorption spectra reveals that pPDI has no "good wavelength band" to achieve de-excitation without substantial excitation to higher states. Nevertheless, when the (photoinduced oxidation) bleaching pathways from the higher states or the triplet state are blocked by working under a nitrogen atmosphere, improved STED images of pPDI can be recorded, as shown in Figure S4. Like the $1 \mathrm{MHz}$ repetition rate experiment, this finding indicates the participation of long-lived (triplet) states in bleaching while also underscoring the relevance of avoiding pumping to higher states in STED microscopy.

In conclusion, our work provides a rationale for the suitability of dyes for STED microscopy. We have demonstrated that the excited-state properties are essential for transiently keeping the fluorophore dark by stimulated emission. Dyes for which the $S_{1} \rightarrow$ $\mathrm{S}_{n}$ absorption spectrum is shifted with respect to its stimulatedemission counterpart satisfy the prerequisite for excellent performance in STED microscopy. For a given fluorophore, the location of the excited-state absorption band is difficult to predict, but for many dyes, the excited-state properties have been published. For example, a dye with little $S_{1} \rightarrow S_{n}$ absorption is the red-emitting dye Atto647N, which to date has been the most popular dye for STED at $\sim 750 \mathrm{~nm}$. Hence, our investigations suggest suitable fluorophores and guide the optimization of the experimental settings. Moreover, they pinpoint a chief criterion, namely, spectral decoupling of the stimulated-emission and $\mathrm{S}_{1} \rightarrow \mathrm{S}_{n}$ bands, for designing and selecting dyes for a non-diffraction-limited far-field fluorescence microscopy that is based just on molecular state transitions.

Acknowledgment. We acknowledge D. Schwarzer, E. Rittweger, and A. Walter (all of Max Planck Institute for Biophysical Chemistry) for valuable discussions. Financial support from the "Fonds voor Wetenschappelijk Onderzoek FWO" (Grant G.0366.06), the K. U. Leuven Research Fund (GOA 2006/2, Center of Excellence CECAT, CREA2007), the Flemish Government (longterm structural funding-Methusalem), and the Federal Science Policy of Belgium (IAP-VI/27) is gratefully acknowledged. P. D. thanks the FWO for a postdoctoral fellowship. This work, as part of the European Science Foundation EUROCORES Program SONS, was supported by funds from the FWO and the E.C. Sixth Framework Program (ERAS-CT-2003-980409).
Supporting Information Available: Structures and complete chemical names of pPDI and pTDI, sample preparation for STED microscopy, details of the experimental setup for CW STED microscopy, STED images of pPDI with the $76 \mathrm{MHz}$ system, CW STED images of pPDI under nitrogen, pulse-energy dependence of STED resolution, and a reference for triplet absorption. This material is available free of charge via the Internet at http://pubs.acs.org.

\section{References}

(1) (a) Abbe, E. Arch. Mikrosk. Anat. 1873, 9, 413. (b) Lord Rayleigh, Philos. Mag., Ser. $5 \mathbf{1 8 9 6 , 4 2 , 1 6 7 . ~}$

(2) Webb, R. H. Rep. Prog. Phvs. 1996, 59, 427.

(3) (a) Weiss, S. Nat. Struct. Biol. 2000, 7, 724. (b) Moerner, W. E. J. Phys. Chem. B 2002, 106, 910. (c) Tinnefeld, P.; Sauer, M. Angew. Chem., Int. Ed. 2005, 44, 2642.

(4) (a) Roeffaers, M. B. J.; Sels, B. F.; Uji-i, H.; De Schryver, F. C.; Jacobs, P. A.; De Vos, D. E.; Hofkens, J. Nature 2006, 439, 572. (b) Dedecker, P.; Muls, B.; Deres, A.; Uji-i, H.; Hotta, J.; Sliwa, M.; Soumillion, J.-P.; Müllen, K.; Enderlein, J.; Hofkens, J. Adv. Mater. 2009, 21, 1079.

(5) Hell, S. W.; Wichmann, J. Opt. Lett. 1994, 19, 780.

(6) Willig, K. I.; Harke, B.; Medda, R.; Hell, S. W. Nat. Methods 2007, 4, 915 .

(7) Betzig, E.; Patterson, G. H.; Sougrat, R.; Lindwasser, O. W.; Olenych, S.; Bonifacino, J. S.; Davidson, M. W.; Lippincott-Schwartz, J.; Hess, H. F. Science 2006, 313, 1642.

(8) Hess, S. T.; Girirajan, T. P. K.; Mason, M. D. Biophys. J. 2006, 91, 4258.

(9) Rust, M. J.; Bates, M.; Zhuang, X. Nat. Methods 2006, 3, 793.

(10) Heilemann, M.; van de Linde, S.; Schuttpelz, M.; Kasper, R.; Seefeldt, B.; Mukherjee, A.; Tinnefeld, P.; Sauer, M. Angew. Chem., Int. Ed. 2008, 47, 6172.

(11) (a) Heilemann, M.; Dedecker, P.; Hofkens, J.; Sauer, M. Laser Photonics Rev. 2009, 3, 180. (b) Dedecker, P.; Hotta, J.; Flors, C.; Sliwa, M.; Uji-i, H.; Roeffaers, M. B. J.; Ando, R.; Mizuno, H.; Miyawaki, A.; Hofkens, J. J. Am. Chem. Soc. 2007, 129, 16132. (c) Flors, C.; Hotta, J.; Uji-i, H.; Dedecker, P.; Ando, R.; Mizuno, H.; Miyawaki, A.; Hofkens, J. J. Am. Chem. Soc. 2007, 129, 13970. (d) Fujita, K.; Kobayashi, M.; Kawano, S.; Yamanaka, M.; Kawata, S. Phys. Rev. Lett. 2007, 99, 228105.

(12) (a) Keller, J.; Schönle, A.; Hell, S. W. Opt. Express 2007, 15, 3361. (b) Dedecker, P.; Muls, B.; Hofkens, J.; Enderlein, J.; Hotta, J. Opt. Express 2007, 15, 3372 .

(13) Rittweger, E.; Rankin, B. R.; Westphal, V.; Hell, S. W. Chem. Phys. Lett. 2007, 442, 483

(14) Song, L.; Varmaa, C. A.; Verhoevena, J. W.; Tankea, H. J. Biophys. J. 1996, 70, 2959

(15) Donnert, G.; Keller, J.; Medda, R.; Andrei, M. A.; Rizzoli, S. O.; Luehrmann, R.; Jahn, R.; Eggeling, C.; Hell, S. W. Proc. Natl. Acad. Sci. U.S.A. 2006, 103, 11440 .

(16) Tinnefeld, P.; Hofkens, J.; Herten, D. P.; Masuo, S.; Vosch, T.; Cotlet, M.; Habuchi, S.; Müllen, K.; De Schryver, F. C.; Sauer, M. ChemPhysChem 2004, 5, 1786.

(17) (a) Uji-i, H.; Melnikov, S. M.; Deres, A.; Bergamini, G.; De Schryver, F.; Herrmann, A.; Müllen, K.; Enderlein, J.; Hofkens, J. Polvmer 2006, 47, 2511. (b) Braeken, E.; De Cremer, G.; Marsal, P.; Pepe, G.; Müllen, K.; Vallee, R. A. L. J. Am. Chem. Soc. 2009, 131, 12201. (c) Kirstein, J.; Platschek, B.; Jung, C.; Brown, R.; Bein, T.; Brauchle, C. Nat. Mater. 2007, 6,303 .

(18) Herrmann, A.; Müllen, K. Chem. Lett. 2006, 35, 978.

(19) Wildanger, D.; Rittweger, E.; Kastrup, L.; Hell, S. W. Opt. Express 2008, $16,9614$.

(20) Donnert, G.; Eggeling, C.; Hell, S. W. Photochem. Photobiol. Sci. 2009, 8,481

(21) Fron, E.; Pilot, R.; Schweitzer, G.; Qu, J.; Herrmann, A.; Müllen, K.; Hofkens, J.; Van der Auweraer, M.; De Schryver, F. C. Photochem. Photobiol. Sci. 2008, 7, 597.

JA100079W 